\title{
Sırt Çantası Problemi İçin Yeni Önerilmiş İkili Krill Sürüsü Algoritması
}

\author{
Emine Baş $^{1 *}$ \\ 1* Selçuk Üniversitesi, Kulu Meslek Yüksekokulu, Bilgisayar Teknolojileri Bölümü, Konya, Türkiye, (ORCID: 0000-0003-4322-6010), emineozcan@selcuk.edu.tr
} (International Conference on Design, Research and Development (RDCONF) 2021 - 15-18 December 2021)

(DOI: $10.31590 /$ ejosat.1039616)

\begin{abstract}
ATIF/REFERENCE: Baş, E., (2021). Sırt Çantası Problemi İçin Yeni Önerilmiş İkili Krill Sürüsü Algoritması. Avrupa Bilim ve
\end{abstract} Teknoloji Dergisi, (32), 113-117.

Öz

Sürü davranışı, benzer büyüklükteki ve aynı yerde bir araya getirilen veya aynı yönde göç eden hayvanların ortak davranışıdır. Sürü davranışlarının taklit edilmesiyle oluşturulmuş birçok algoritma literatürde mevcuttur. Krill algoritmasıda (KH) bu tarz algoritmalardan birisidir. KH algoritması, Krill sürüsünün yaşam tarzını incelemiştir. Krill, okyanus ekosisteminin yapı taşı olarak kullanılır. Krill yaratıkları her zaman büyük sürüler halinde yaşar. Krill sürülerinin seyahat etmesinin iki nedeni vardır. Birincisi diğer canlılar tarafından yenmeden hayatta kalmak ve krill canlı sürünün yoğunluğunu arttırmak, diğer amaç ise daha kolay avlanmaktır. Bu çalışmada KH algoritması ikili optimizasyon problemlerini çözebilecek şekilde tekrardan güncellenmiştir. Uygulamalar ikili bir optimizasyon problemi olan sırt çantası problemi üzerinde test edilmiştir.

Anahtar Kelimeler: Krill, ikili optimizasyon, Sırt çantası problemi.

\section{Newly Proposed Binary Krill Swarm Algorithm for Backpacking Problem}

\begin{abstract}
Herd behavior is the collective behavior of animals of similar size that gather in the same place or migrate in the same direction. Many algorithms created by imitating swarm behaviors are available in the literature. Krill algorithm (KH) is one of such algorithms. The KH algorithm studied the lifestyle of the Krill herd. Krill is used as the building block of the ocean ecosystem. Krill creatures always live in large herds. There are two reasons why krill swarms travel. The first is to survive without being eaten by other creatures and to increase the density of the krill living herd, and the other is to be hunted more easily. In this study, the KH algorithm has been updated again to solve the binary optimization problems. The applications are tested on the backpack problem, which is a binary optimization problem.
\end{abstract}

Keywords: Krill, Binary optimization, Backpack problem.

\footnotetext{
*Sorumlu Yazar: emineozcan@selcuk.edu.tr
} 


\section{Giriş}

Günümüzde birçok araştırmacı çözülmesi zor olan gerçek dünya problemlerine çözüm aramaktadır. Bu tarz zor problemler için yeni ve eski birçok yöntem önerilmiştir (İhsan ve Ülker, 2017). Sezgisel algoritmalar çözülmesi zor problemleri çözmek için önerilmiş yeni yöntemlerden birisidir. Sezgisel algoritmalar doğadaki çeşitli hayvanların yaşamlarının modellenmesi ile oluşturulmuş yeni algoritmalardır. Krill algoritması $(\mathrm{KH})$ da sezgisel bir algoritmadır. Gandomi ve Alavi tarafından 2012 de önerilmiştir (Gandomi ve Alavi, 2012). Krill sürülerinin yaşam şeklinin taklit edilmesi ile oluşturulmuştur. Kril sürüleri sürüler halinde yaşar ve bir liderleri vardır. Krill sürüsü, besin zincirinin önemli bir yapı taşıdır. Krill, balina, fok, balık, kalamar, penguenler ve deniz kuşları gibi birçok hayvanın besin kaynağıdır. Kril canlılarının ortalama uzunluğu 5-10 cm, ortalama ömürleri ise 2 ila 6 yıldır. Kril kabuklarından dolayı karidese benzer. Siyah iri gözler ve şeffaf vücuttan oluşan vücut yapıları sayesinde sindirim sistemleri dışarıdan görülebilir.

Optimizasyon kelime anlamıla bir gerçel fonksiyonu minimize ya da maksimize etmek amacı ile gerçek ya da tam sayı değerlerini tanımlı bir aralıkta seçip fonksiyona yerleştirerek sistematik olarak bir problemi incelemek ya da çözmek işlemlerini ifade eder. Optimizasyon problemleri iki gruba ayrılması mümkündür. Bunlar sürekli optimizasyon ve ayrık optimizasyondur. Ayrık optimizasyon problemlerini arama uzayındaki değerlerin integer veya ikili olması durumlarına göre de alt gruba ayırmak mümkündür. Optimizasyon problemlerinde arama uzayı her zaman integer değerlerden oluşmayabilir. İkili değerler şeklinde oluşturulmuş arama uzaylarıda ayrık optimizasyon problemlerini çözmek için kullanılmaktadır. Bu tarz optimizasyon problemlerine İkili Optimizasyon Problemleri (İOP) denmektedir (Baş ve Ülker, 2020). Literatürde birçok İOP türü problem mevcuttur. 0-1 Sirt çantası problemi de bu tarz problemlerden birisidir. 0-1 Sırt çantası probleminde arama uzayında sırt çantasına konacak $n$ adet ürün ile bilgi tutulmaktadır. Arama uzayında 0 değeri ilgili ürünün çantaya konmayacağını, 1 değeri ise ilgili ürünün çantaya konacağını göstermektedir (Hakli, 2020).

\section{Materyal ve Metot}

\subsection{Krill Sürüsü Algoritması (KH)}

Krill Fransızca bir kelimedir ve "bebek balık" anlamına gelir. Krill, dünyadaki en yaygın kabuklulardan biridir. Aynı zamanda Krill dünyasındaki çok hücreli hayvan türleri arasında en büyüğüdür. Krill sürüsü, besin zincirinin önemli bir yapı taşıdır. Krill, balina, fok, balık, kalamar, penguenler ve deniz kuşları gibi birçok hayvanın besin kaynağıdır. Krill canlılarının ortalama uzunluğu 5-10 cm, ortalama ömürleri ise 2 ila 6 yıldır. Krill kabuklarından dolayı karidese benzer. Siyah iri gözler ve şeffaf vücuttan oluşan vücut yapıları sayesinde sindirim sistemleri dışarıdan görülebilir. Şekil 1 de krill canlısının vücut yapısı gösterilmiştir

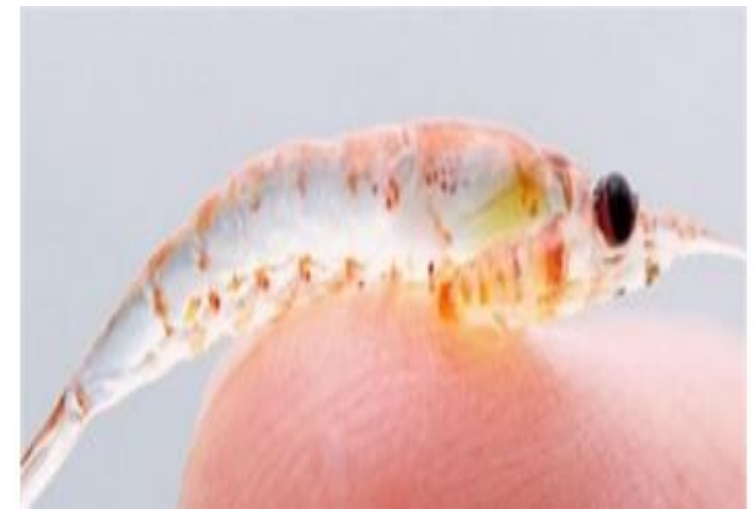

Şekil 1. Krill canlısının vücut yapısı

\subsubsection{Krill sürülerinin sürü davranışı}

Krill sürüsü ekosistemimizde önemli rollere sahiptir. Bu deniz hayvanı, $91 \mathrm{~m}$ veya $304 \mathrm{~m}$ derinlikte dünyadaki tüm okyanuslarda yaşayabilir. Canlıların okyanuslardaki dağılımı yaşadıkları bölgedeki suyun sıcaklığına göre değişir. Krill sürüleri sürüler halinde hareket eder ve onları koruyan bir yapı oluşturur. Krill canlılarının birlikte hareket ederek oluşturduğu kalabalık sürülerin amacı diğer canlılardan sürünün yenmesini zorlaştırmaktadır.

\subsubsection{Krill sürüsünün Lagrange modeli}

Krill sürüsünün bir lideri vardır. Bir lider olarak Krill, canlı besleme, üreme ve diğer canlılardan korunma gibi çiftlik hayvanlarının davranışları hakkında kararlar alır. Alınan kararlar, sürüdeki diğer Krill sürüsü tarafından sorgulanmadan uygulanır. Algoritmayı modellemek için aşağıdaki denklemler kullanılır. Denklem 1, 2 ve denklem 3'de kril yaratıklarının hareketleri formüle edilmiştir.

$\frac{\mathrm{dx}_{\mathrm{i}}}{\mathrm{dt}}=\mathrm{N}_{\mathrm{i}}+\mathrm{F}_{\mathrm{i}}+\mathrm{D}_{\mathrm{i}}$

$\mathrm{N}_{\mathrm{i}}^{\text {new }}=\mathrm{N}^{\text {max }} \mathrm{a}_{\mathrm{i}}+\mathrm{w}_{\mathrm{n}} \mathrm{N}_{\mathrm{i}}^{\text {old }}$

$\mathrm{a}_{\mathrm{i}}=\mathrm{a}_{\mathrm{i}}^{\text {local }}+\mathrm{a}_{\mathrm{i}}^{\text {target }}$

Burada $N$ hızdır ve $0.01(\mathrm{~m} / \mathrm{s})$ olarak alınır. $a_{\mathrm{i}}$ hareket yönü, $N_{i}^{\text {old }}$ son konum, $a_{i}^{\text {local }}$ komşunun etkileri ve $a_{i}^{\text {target }}$ krill sürüleri tarafindan seçilen hedefi gösterir. Denklem 4, 5 ve denklem 6'da $a_{i}^{\text {local }}$ hesaplamaları gösterilmiştir.

$\mathrm{a}_{\mathrm{i}}^{\text {local }}=\sum \widehat{\mathrm{K}}_{\mathrm{i}, \mathrm{j}} \widehat{\mathrm{X}}_{\mathrm{i}, \mathrm{j}}$

$\widehat{X}_{i, j}=\frac{x_{j}-x_{i}}{\left\|x_{j}-x_{i}\right\|+\varepsilon}$

$\mathrm{K}_{\mathrm{i}, \mathrm{j}}=\frac{\mathrm{K}_{\mathrm{i}}-\mathrm{K}_{\mathrm{j}}}{\mathrm{K}^{\mathrm{worst}}-\mathrm{K}^{\text {best }}}$

Krill canlıların uygunluk değerleri hesaplanmış ve en iyi optimum değer $K^{\text {best }}$, en kötü optimum değer $K^{\text {worst }}$ şeklinde gösterilmiştir. Krill yaratıkların amaç fonksiyon değeri $K_{i}$, komşu krill yaratıkların amaç fonksiyon değeri $K_{j}$ parametreleriyle gösterilmiştir. $N_{N}$, toplam komşu sayısını, kril sürülerinin 
konumunu $X$ temsil etmektedir. Şekil 2 ye göre algılama mesafesi (sensing distance) komşu seçimine göre yapılmaktadır. Komşu seçimi $d_{s}$ ile gösterilmiştir. Denklem 7 de gösterilmiştir.

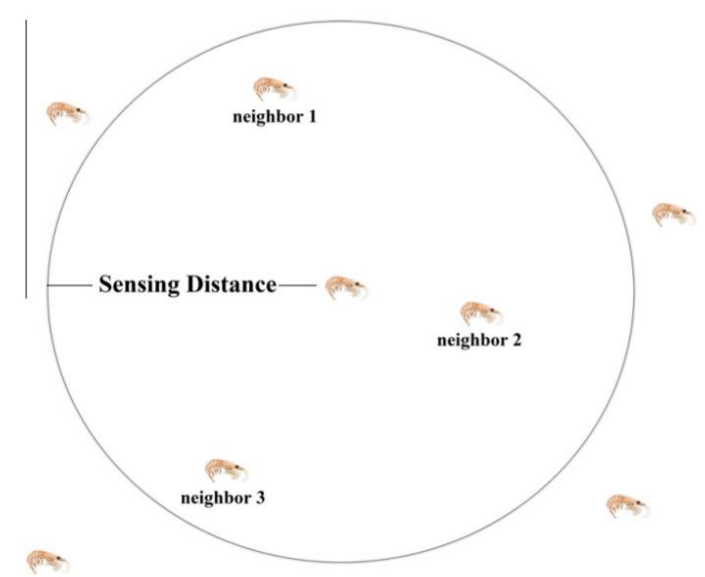

Şekil 2. Krill sürüsü etrafındaki komşuların tespiti

$\mathrm{d}_{\mathrm{s}, \mathrm{i}}=\frac{1}{5 \mathrm{~N}} \sum_{\mathrm{J}=1}^{\mathrm{N}}\left\|\mathrm{X}_{\mathrm{i}}-\mathrm{X}_{\mathrm{j}}\right\|$

$d_{s, i}$ algılanan mesafeyi gösterir, $N$ toplam kril birey sayısını verir. Denklem 8'e göre, $\mathrm{d}_{\mathrm{s}}$ 'den küçük iki Krill sürüsü arasındaki mesafe, Krill bireylerinin komşu olduğu sonucunu gösterir. [0 1]'deki rasgele sayı, $I$ döngü sayısını ve $I_{\max }$ maksimum döngü sayısını göstermektedir.

$\mathrm{a}_{\mathrm{i}}^{\text {target }}=\mathrm{C}^{\text {best }} \widehat{\mathrm{K}}_{\mathrm{i} \text {,best }} \widehat{\mathrm{X}}_{\mathrm{i} \text {,best }}$

$\mathrm{C}^{\text {best }}=2\left(\right.$ rand $\left.+\frac{\mathrm{I}}{\mathrm{I}_{\max }}\right)$

\subsubsection{Arama Hareketi}

Yiyecek arama sırasında iki faaliyet gerçekleştirilir. Krill yaratıkları öncelikle yiyeceğin yerini keşfetmeyi amaçlar. Diğer süreç deneyimdir. Bu davranışlar aşağıdaki denklemlerde formüle edilmiştir.

$$
\begin{aligned}
& F_{i}=V_{f} \beta_{i}+W_{f} F_{i}^{\text {old }} \\
& \beta_{i}=\beta_{i}{ }^{\text {food }}+\beta_{\dot{I}}^{\text {best }} \\
& x_{i}^{\text {food }}=\frac{\sum_{i=1}^{N}\left(1 / K_{i}\right) X_{i}}{\sum_{i=1}^{N}\left(1 / K_{i}\right)} \\
& \beta_{i}^{\text {food }}=C^{\text {food }} \widehat{K}_{i, \text { food }} \widehat{X}_{i, f o o d} \\
& C^{\text {food }}=2\left(1-\frac{I}{I_{\text {max }}}\right) \\
& \beta_{i}^{\text {best }}=\widehat{K}_{i, \text { best }} \widehat{X}_{i, \text { best }}
\end{aligned}
$$

\subsubsection{Fiziksel Difüzyon}

Kril sürüsünün bireylerinin sudaki dağılımı rastgeledir. Kril sürüsü, hareket yönünü rastgele seçer ve hızı, maksimum yayılma hızı olarak kabul edilir. Rastgele bir sürecin fiziksel yayılımı aşağıdaki gibi formüle edilir. Burada maksimum yayılma hızı $D^{\max } \in[0.002,0.010]\left(\mathrm{m} / \mathrm{s}^{-1}\right)$ ve $\delta$ rastgele yönlü vektör ve değerler $[-1,1]$ aralığındadır.

$\mathrm{D}_{\mathrm{i}}=\mathrm{D}^{\max }\left(1-\frac{\mathrm{I}}{\mathrm{I}_{\max }}\right) \delta$

Denklemdeki $D^{\max }$, maksimum difüzyon hızıdır ve $\delta$, rastgele seçilen yönün parametresidir. $\delta$ parametresi $[-1,1]$ 'den rastgele seçilir. Krill bireylerinin difüzyon hızı için önerilen maksimum aralık $D^{\max } \in[0,002,0,010]\left(\mathrm{m} / \mathrm{s}^{-1}\right)$ ve rastgele bir sayı olarak seçilir.

\subsubsection{KH Algoritmasının Hareket Süreci}

$t$ ve $t+\Delta t$ zaman esnasında bir krill bireyinin konum vektörü denklem 17 ve 18 de gösterilmiştir. NV toplam değişken sayısı, $\mathrm{LB}_{\mathrm{j}}$ ve $\mathrm{UB}_{\mathrm{j}}$ parametereleri ise $j$ değişkenin üst ve alt limitleri, $\mathrm{C}_{t}$ ise bir sabit değeri gösterir. $C_{t}$ değeri 0.5 olarak çalışmada alınmıştır.

$D_{i}(t+\Delta t)=X_{i}(t)+\Delta t \frac{d X_{i}}{d t}$

$\Delta \mathrm{t}=\mathrm{C}_{\mathrm{t}} \sum_{\mathrm{j}=1}^{\mathrm{NV}} \mathrm{UB}_{\mathrm{j}}-\mathrm{LB}_{\mathrm{j}}$

\section{2. İkili Krill Sürüsü Algoritması (İKH)}

Orijinal Krill sürüsü algoritmasında arama uzayı sürekli değerlerden oluşmaktadır. Surt çantası probleminde ise arama uzayının ikili değerlere çevrilmesi gerekmektedir. Bu arama uzayındaki sürekli değerler denklem 19 a göre ikili değerlere çevrilmiştir. Çevrilen ikili arama uzayı sırt çantası probleminde kullanılmıştır. Diğer çalışma adımları krill sürüsü algoritması ile aynıdır. Böylece İkili Krill sürüsü algoritması (İKH) önerilmiştir.

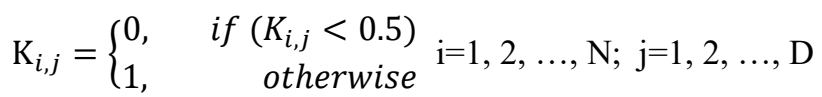

\subsection{0-1 Sırt Çantası Problemi}

Tipik bir NP-zor problemi olan 0-1 sırt çantası problem şu şekilde tanımlanmaktadır: Elimizde $N$ adet nesne olduğunu varsayalım. $W$ değeri sırt çantasının kapasitesini göstersin. Her $i$ nesnenin bir ağırlık $\left(\mathrm{w}_{\mathrm{i}}\right)$ ve bir kar değeri $\left(\mathrm{p}_{\mathrm{i}}\right)$ mevcuttur. Asıl amaç, sırt çantasının kapasitesinin sınırını aşmadan sırt çantasının toplam kârını maksimize etmektir. Problemin matematiksel modeli çok basittir ve şu şekilde formüle edilmiştir: 
$\operatorname{Maximize}\left(f(x)=\sum_{i=1}^{N} x_{i} p_{i}\right.$

subject to $\sum_{i=1}^{N} x_{i} w_{i} \leq W \quad x_{i} \in\{0,1\}, i=1,2,3, \ldots, N$

$x_{i}$ (ikili değişken), $i$ nesnesinin sırt çantasında olup olmayacağını temsil etmektedir.

\section{Araştırma Sonuçları ve Tartışma}

Algoritma kodlamaları Matlab R2014a üzerinde kodlanmıştır. Uygulamalar işlemcisi intel Core i5 $1.19 \mathrm{Ghz}$, ram değeri $12 \mathrm{~Gb}$ olan işletim sistemi windows 10 olan bir makine üzerinde çalıştırılmıştır. 25 farklı $0-1$ sırt çantası veri seti uygulamalarda kullanılmıştır. İkili KH nin performansı en iyi, en kötü, ortalama ve standart sapma hesaplamaları yapılarak gösterilmiştir. Her bir uygulama 20 bağımsız çalışma şeklinde değerlendirilmiştir. Uygulamalarda kullanılan parametre değerleri tablo 1 de gösterilmiştir. Sonuçlar tablolar 2-6 da gösterilmiştir.

Tablo 7 de İkili Denge Optimizasyonu Algoritması (BEO) (Abdel-Baset ve ark., 2021) ve İkili KH algoritmas1 karşılaştırması için kullanılan parametreler sunulmuştur. Tablo 8 de BEO ve İKH algoritma sonuçları gösterilmiştir. Sonuçlara göre ilk beş veri setinde her iki algoritmada üstün performans göstermiştir. P6-P25 arasında kullanılan veri setlerinde ise BEO daha üstün başarı göstermiştir.

Tablo 1. Parametre ayarlart

\begin{tabular}{|c|c|c|}
\hline Algoritma & Parametre & Değer \\
\hline \multirow{4}{*}{} & $\mathrm{C}_{\mathrm{t}}$ & 0.5 \\
\cline { 2 - 3 } & $\mathrm{Dmax}$ & 0.005 \\
\cline { 2 - 3 } & $\mathrm{Nmax}$ & 0.01 \\
\cline { 2 - 3 } & $\mathrm{Vf}$ & 0.02 \\
\cline { 2 - 3 } & Populasyon say1s1 & 40 \\
\cline { 2 - 3 } & Boyut & $8,12,16,20,24$ \\
\cline { 2 - 3 } & Maksimum döngü & $1000(\mathrm{P} 1-\mathrm{P} 10) \mathrm{ve}$ \\
& & $5000(\mathrm{P} 11-\mathrm{P} 25)$ \\
\hline
\end{tabular}

Tablo 2. Sırt çantası problemi için IKH sonuçları (Boyut $=8)$

\begin{tabular}{|c|c|c|c|c|c|}
\hline $\begin{array}{c}\text { Problem } \\
\text { No }\end{array}$ & Optimum & En iyi & En kötü & Ortalama & $\begin{array}{c}\text { Standart } \\
\text { Sapma }\end{array}$ \\
\hline$P 1$ & 3924400 & 3924400 & 3924400 & 3924400 & 0 \\
\hline$P 2$ & 3813669 & 3813669 & 3813669 & 3813669 & 0 \\
\hline$P 3$ & 3347452 & 3347452 & 3347452 & 3347452 & 0 \\
\hline$P 4$ & 4187707 & 4187707 & 4187707 & 4187707 & 0 \\
\hline$P 5$ & 4955555 & 4955555 & 4955555 & 4955555 & 0 \\
\hline
\end{tabular}

Tablo 3. Sirt çantası problemi için IKH sonuçları (Boyut=12)

\begin{tabular}{|c|c|c|c|c|c|}
\hline $\begin{array}{c}\text { Proble } \\
m \text { No }\end{array}$ & Optimum & En iyi & En kötü & $\begin{array}{c}\text { Ortalam } \\
a\end{array}$ & $\begin{array}{c}\text { Standart } \\
\text { Sapma }\end{array}$ \\
\hline$P 6$ & 5688887 & 5688887 & 5644340 & 5655456 & 18843,76 \\
\hline$P 7$ & 6498597 & 6498597 & 6409596 & 6470450 & 37269,68 \\
\hline$P 8$ & 5170626 & 5170626 & 5115388 & 5162881 & 18633,48 \\
\hline
\end{tabular}

\begin{tabular}{|c|c|c|c|c|c|}
\hline$P 9$ & 6992404 & 6992404 & 6919995 & 6959317 & 28257,9 \\
\hline$P 10$ & 5337472 & 5337472 & 5194308 & 5289302 & 47376,2 \\
\hline
\end{tabular}

Tablo 4. Sırt çantası problemi için IKKH sonuçları (Boyut=16)

\begin{tabular}{|c|c|c|c|c|c|}
\hline $\begin{array}{c}\text { Problem } \\
\text { No }\end{array}$ & Optimum & En iyi & En kötü & Ortalama & $\begin{array}{c}\text { Standart } \\
\text { Sapma }\end{array}$ \\
\hline P11 & 7850983 & 7850983 & 7664852 & 7746832 & 49912,43 \\
\hline P12 & 9352998 & 9352998 & 9083443 & 9214199 & 71764,56 \\
\hline P13 & 9151147 & 9151147 & 8907854 & 9012090 & 58006,36 \\
\hline$P 14$ & 9348889 & 9348889 & 9129802 & 9244964 & 57138,36 \\
\hline P15 & 7769117 & 7746722 & 7544868 & 7661586 & 51871 \\
\hline
\end{tabular}

Tablo 5. Sırt çantası problemi için IKKH sonuçları (Boyut=20)

\begin{tabular}{|c|c|c|c|c|c|}
\hline $\begin{array}{c}\text { Problem } \\
\text { No }\end{array}$ & Optimum & En iyi & En kötü & Ortalama & $\begin{array}{c}\text { Standart } \\
\text { Sapma }\end{array}$ \\
\hline$P 16$ & 10727049 & 10690960 & 10375652 & 10536557 & 84051,8 \\
\hline$P 17$ & 9818261 & 9729415 & 9553463 & 9616044 & 46027,2 \\
\hline$P 18$ & 10714023 & 10642072 & 10312238 & 10485805 & 80991,9 \\
\hline$P 19$ & 8929156 & 8885666 & 8658254 & 8763080 & 57423,9 \\
\hline$P 20$ & 9357969 & 9357969 & 9139383 & 9250440 & 61147,1 \\
\hline
\end{tabular}

Tablo 6. Sirt çantası problemi için IKH sonuçları (Boyut=24)

\begin{tabular}{|c|c|c|c|c|c|}
\hline $\begin{array}{c}\text { Problem } \\
\text { No }\end{array}$ & Optimum & En iyi & En kötï & Ortalama & $\begin{array}{c}\text { Standart } \\
\text { Sapma }\end{array}$ \\
\hline$P 21$ & 13549094 & 13399433 & 13078908 & 13233534 & 85529,76 \\
\hline$P 22$ & 12233713 & 12095195 & 11882538 & 11983099 & 55861,83 \\
\hline$P 23$ & 12448780 & 12388310 & 12079794 & 12212581 & 69715,3 \\
\hline$P 24$ & 11815315 & 11715017 & 11420592 & 11570009 & 71676,6 \\
\hline$P 25$ & 13940099 & 13836922 & 13485098 & 13666388 & 93966,85 \\
\hline
\end{tabular}

Tablo 7. Sirt çantası problemi için IKH ve BEO için parametre ayarlart

\begin{tabular}{|c|c|c|}
\hline Algoritma & Parametre & Değer \\
\hline \multirow{4}{*}{ IKH } & $\mathrm{C}_{\mathrm{t}}$ & 0.5 \\
\cline { 2 - 3 } & $\mathrm{Dmax}$ & 0.005 \\
\cline { 2 - 3 } & $\mathrm{Nmax}$ & 0.01 \\
\cline { 2 - 3 } & $\mathrm{Vf}$ & 0.02 \\
\cline { 2 - 3 } & Populasyon sayıs1 & 40 \\
\cline { 2 - 3 } & Boyut & $8,12,16,20,24$ \\
\cline { 2 - 3 } & Maksimum döngü & $1000(\mathrm{P} 1-\mathrm{P} 10) \mathrm{ve}$ \\
& & $5000(\mathrm{P} 11-\mathrm{P} 25)$ \\
\hline \multirow{4}{*}{ BEO } & Populasyon sayıs1 & 20 \\
\cline { 2 - 3 } & Boyut & $8,12,16,20,24$ \\
\cline { 2 - 3 } & Maksimum döngü & 5000 \\
\cline { 2 - 3 } & $\mathrm{a}_{1}$ ve $\mathrm{a}_{2}$ & 3 ve 1 dir. \\
\hline
\end{tabular}

Tablo 8. Sirt çantası problemi için IKH ve BEO karşılaştırma sonuçları

\begin{tabular}{|c|c|c|c|c|}
\hline \multirow{2}{*}{$\begin{array}{c}\text { Problem } \\
\text { No }\end{array}$} & \multicolumn{2}{|c|}{ IKH } & \multicolumn{2}{c|}{ BEO } \\
\cline { 2 - 5 } & Ortalama & $\begin{array}{c}\text { Standart } \\
\text { Sapma }\end{array}$ & Ortalama & $\begin{array}{c}\text { Standart } \\
\text { Sapma }\end{array}$ \\
\hline$P 1$ & 3924400 & 0 & 3924400 & 0 \\
\hline$P 2$ & 3813669 & 0 & 3813669 & 0 \\
\hline
\end{tabular}




\begin{tabular}{|c|c|c|c|c|}
\hline$P 3$ & 3347452 & 0 & 3347452 & 0 \\
\hline$P 4$ & 4187707 & 0 & 4187707 & 0 \\
\hline$P 5$ & 4955555 & 0 & 4955555 & 0 \\
\hline$P 6$ & 5655456 & 18843,76 & 5688887 & 0 \\
\hline$P 7$ & 6470450 & 37269,68 & 6498597 & 0 \\
\hline$P 8$ & 5162881 & 18633,48 & 5170626 & 0 \\
\hline$P 9$ & 6959317 & 28257,9 & 6992404 & 0 \\
\hline$P 10$ & 5289302 & 47376,2 & 5337472 & 0 \\
\hline$P 11$ & 7746832 & 49912,43 & 7850983 & 0 \\
\hline$P 12$ & 9214199 & 71764,56 & 9352998 & 0 \\
\hline$P 13$ & 9012090 & 58006,36 & 9151147 & 0 \\
\hline$P 14$ & 9244964 & 57138,36 & 9348889 & 0 \\
\hline$P 15$ & 7661586 & 51871 & 7769117 & 0 \\
\hline$P 16$ & 10536557 & 84051,8 & 10727049 & 0 \\
\hline$P 17$ & 9616044 & 46027,2 & 9818261 & 0 \\
\hline$P 18$ & 10485805 & 80991,9 & 10714023 & 0 \\
\hline$P 19$ & 8763080 & 57423,9 & 8929156 & 0 \\
\hline$P 20$ & 9250440 & 61147,1 & 9357969 & 0 \\
\hline$P 21$ & 13233534 & 85529,76 & 13549094 & 0 \\
\hline$P 22$ & 11983099 & 55861,83 & 12233713 & 0 \\
\hline$P 23$ & 12212581 & 69715,3 & 12448780 & 0 \\
\hline$P 24$ & 11570009 & 71676,6 & 11814367 & 1147,262 \\
\hline$P 25$ & 13666388 & 93966,85 & 13940099 & 0 \\
\hline & & & & \\
\hline
\end{tabular}

düşük, orta ve yüksek ölçekli veri seti kullanılmıştır. İKH nin başarıs bu veri setlerinde gösterilmiştir. İKH nin başarısı literatürde yeni önerilmiş BEO algoritması ile de karşılaştırılmıştır. Sonuçlar göstermiştir ki İKH nin local ve global araması geliştirilmelidir.

Geleceğe dair çalışmalarda İKH nin arama yeteneğinin farklı teknikler kullanılarak geliştirilmesi düşünülmektedir. İKH nin ayrk farklı optimizasyon problemlerinde başarısı da gösterilecektir.

\section{Kaynakça}

Abdel-Baset, M., Mohamed, R., Mirjalili, S., 2021, A Binary Equilibrium Optimization Algorithm for $0-1$ Knapsack Problems, Computers \& Industrial Engineering 151 (20219 106946.

İhsan, A. A., Ulker, E., 2017, Development of the Egyptian Vulture Optimization Algorithm with the Nearest or Farest Neighborhood Methods, ISMSIT2017, Tokat - Turkey, 179 182.

Gandomi, A. H., \& Alavi, A. H., Krill herd: A new bio-inspired optimization algorithm, Communications in Nonlinear Science and Numerical Simulation, 2012; 17(12): 4831 4845.

Hakli, H., 2020, BinEHO: a new binary variant based on elephant herding optimization algorithm, Neural Computing and Applications (2020) 32:16971-16991.

Krill algoritması $(\mathrm{KH})$ sezgisel bir algoritmadır. Krill sürüsü, besin zincirinin önemli bir yapı taşıdır. Krill sürülerinin yaşam şeklinin taklit edilmesi ile KH algoritması oluşturulmuştur. KH ile literatürde çeşitli çalışmalar yapılmış olmasına ragmen ikili şekli önerilmemiştir. $\mathrm{Bu}$ çalışmada $\mathrm{KH}$ algoritmasının ikili şekli önerilmiştir. İkili optitimizasyonda sıklıkla tercih edilen sırt çantası problemi bu çalışmada tercih edilmiştir. Yirmi beş farklı

Baş, E., Ülker, E. (2020). A binary social spider algorithm for uncapacitated facility location problem, Expert Systems with Applications 161, 113618. 